Corresponding author: Iwartman@dom.wustl.edu

(C) 2016 Lu et al. This article is distributed under the terms of the Creative Commons Attribution-NonCommercial License, which permits reuse and redistribution, except for commercial purposes, provided that the original author and source are credited.

Ontology terms: hematological neoplasm, neoplasm of the genitourinary tract

Published by Cold Spring Harbor Laboratory Press

doi: $10.1101 /$ mcs.a000687

\section{A common founding clone with TP53 and PTEN mutations gives rise to a concurrent germ cell tumor and acute megakaryoblastic leukemia}

\author{
Charles Lu, ${ }^{1,5}$ Peter Riedell, ${ }^{2,5}$ Christopher A. Miller, ${ }^{1,3}$ Ian S. Hagemann, ${ }^{4}$ \\ Peter Westervelt, ${ }^{2}$ Bradley A. Ozenberger, ${ }^{1}$ Michelle O'Laughlin, \\ Vincent Magrini, ${ }^{1}$ Ryan T. Demeter, ${ }^{1}$ Eric J. Duncavage, ${ }^{4}$ Malachi Griffith, ${ }^{1}$ \\ Obi L. Griffith, ${ }^{1,2}$ and Lukas D. Wartman ${ }^{1,2}$

\footnotetext{
${ }^{1}$ McDonnell Genome Institute, Washington University School of Medicine, St. Louis, Missouri 63110, USA; ${ }^{2}$ Division of Oncology, Department of Medicine, Washington University School of Medicine, St. Louis, Missouri 63110, USA; ${ }^{3}$ Division of Genomics and Bioinformatics, Department of Medicine, Washington University School of Medicine, St. Louis, Missouri 63110, USA; ${ }^{4}$ Department of Pathology and Immunology,
} \\ Washington University School of Medicine, St. Louis, Missouri 63110, USA
}

\begin{abstract}
We report the findings from a patient who presented with a concurrent mediastinal germ cell tumor (GCT) and acute myeloid leukemia (AML). Bone marrow pathology was consistent with a diagnosis of acute megakaryoblastic leukemia (AML M7), and biopsy of an anterior mediastinal mass was consistent with a nonseminomatous GCT. Prior studies have described associations between hematological malignancies, including AML M7 and nonseminomatous GCTs, and it was recently suggested that a common founding clone initiated both cancers. We performed enhanced exome sequencing on the GCT and the AML M7 from our patient to define the clonal relationship between the two cancers. We found that both samples contained somatic mutations in PTEN (C136R missense) and TP53 (R213 frameshift). The mutations in PTEN and TP53 were present at $\sim 100 \%$ variant allele frequency (VAF) in both tumors. In addition, we detected and validated five other shared somatic mutations. The copy-number analysis of the AML exome data revealed an amplification of Chromosome 12p. We also identified a heterozygous germline variant in FANCA (S858R), which is known to be associated with Fanconi anemia but is of uncertain significance here. In summary, our data not only support a common founding clone for these cancers but also suggest that a specific set of distinct genomic alterations (in PTEN and TP53) underlies the rare association between GCT and AML. This association is likely linked to the treatment resistance and extremely poor outcome of these patients. We cannot resolve the clonal evolution of these tumors given limitations of our data.
\end{abstract}

[Supplemental material is available for this article.]

\section{INTRODUCTION}

Since first recognized in 1985, multiple studies have described the unique and rare association between hematological malignancies and germ cell tumors (GCTs) (DeMent et al.

\footnotetext{
${ }^{5}$ These authors contributed equally to this work.
} 
COLD SPRING HARBOR Molecular Case Studies
A common clone gives rise to a GCT and AML
1985; Nichols et al. 1985, 1990; Woodruff et al. 1995; Hartmann et al. 2000). Most commonly, these cases involve the megakaryocytic lineage of hematopoiesis, frequently resulting in acute megakaryoblastic leukemia (AML M7) and primary mediastinal nonseminomatous GCTs. Initially, the biologic relationship between these two entities was elusive, but the first definitive overview of the syndrome by Nichols et al. (1990) described the significance of an isochromosome 12p in the acute myeloid leukemia (AML) samples from these patients in that it "suggests that the hematologic neoplasm and the mediastinal germ-cell tumor arose from a common progenitor cell" (Hartmann et al. 2000). Isochromosome 12p is the most common chromosomal abnormality identified in GCTs but is exceedingly rare in AML that is not associated with a GCT (Gibas et al. 1986; Hartmann et al. 2000). A potential shared origin of these two malignancies has been further supported by numerous case reports describing a common isochromosome $12 \mathrm{p}$ in the corresponding tumor samples (Chaganti et al. 1989; Landanyi et al. 1990; Ikdahl et al. 2007). These studies laid the groundwork for a potential shared origin of the GCT and AML.

There are two distinct theories that have been generally postulated to explain how these two malignancies could arise from a shared founding clone. In the first, a primitive mesodermal stem cell is the founding clone and harbors the initiating alteration(s) that drives both cancers. The initiating cell would likely arise in early development in the aorta-gonadmesonephros (AGM) region, which is the origin for stem cells that give rise to definitive hematopoiesis (Medvinsky and Dzierzak 1996). For unclear reasons, cells derived from the founding clone would remain in the AGM region but would also be present in the bone marrowand could then give rise to both the GCT in the chest and the AML in the bone marrow. In the alternative theory, the AML is derived directly from cells from the GCT (mostly likely from the yolk sac component of a GCT). Some population of cells from the GCT "seed" (or metastasize to) the bone marrow, which gives rise to the AML (as reviewed in Zhao and Dowell 2012).

The argument for a shared common origin was strengthened by a recent study, which reported the results of targeted massively parallel sequencing of 33 genes, standard cytogenetic/fluorescence in situ hybridization analysis and single-nucleotide polymorphism (SNP) array profiling on samples from a patient with AML M7 and a synchronous mediastinal GCT. The authors identified shared somatic mutations in PTEN (G251V missense) and TP53 (L130P missense), as well as the presence of isochromosome 12p, in both the GCT and AML samples, which further supports that a common founding clone initiated both cancers. However, both the PTEN and TP53 mutations were present at relatively low variant allele frequencies (VAFs) in both samples in that study. For example, in the GCT, the TP53 mutation was only present at a VAF of $5.97 \%$ and the PTEN mutation at a VAF of $15.12 \%$ (with VAFs of $20.35 \%$ and $27.59 \%$ in the AML sample, respectively) (Oshrine et al. 2014). The low VAFs were most likely due to low tumor cell content of the samples that were sequenced, and these data are most consistent with shared mutations present in the founding clone of both cancers.

Herein, we report the results of enhanced exome sequencing from a case of a male patient with a mediastinal GCT and synchronous AML M7. By sequencing minute amounts of tumor DNA, we were able to identify and validate shared somatic mutations in TP53, PTEN, and five other genes (of unknown pathogenic significance). Our data support the hypothesis that these shared alterations are present in the founding clone of the tumors. The copy-number analysis of the AML exome data revealed an amplification of Chromosome 12p (consistent with an isochromosome 12p), which was not detected on standard cytogenetics. We also identified a heterozygous germline variant in FANCA (S858R), which is known to be associated with Fanconi anemia but is of uncertain significance here. This is therefore the second case report to describe the presence of shared TP53 and PTEN somatic mutations in the rare syndrome of concurrent GCT and AML M7, suggesting that these mutations synergize in an important way to contribute to these tumors. 


\section{RESULTS}

\section{Clinical Presentation}

The patient was a 33-yr-old Caucasian male with no significant past medical history who presented with a 1-mo history of generalized weakness, a 40-pound weight loss, and dyspnea on exertion. His initial workup was notable for a platelet count of $5000 / \mu \mathrm{L}$, hemoglobin of $13.1 \mathrm{~g} / \mathrm{dL}$, and a white blood count of $9200 \mathrm{cell} / \mathrm{\mu L}$ with a normal differential. A chest radiograph revealed evidence of a large anterior mediastinal mass; a computed tomography (CT) scan of the chest was performed that showed a $10 \times 7.7 \times 11-\mathrm{cm}$ heterogeneous enhancing anterior mediastinal mass (Fig. 1). Given the suspicion for a GCT in this young man, standard tumor markers were drawn: $\alpha$-fetoprotein (AFP) was $237 \mathrm{ng} / \mathrm{mL}$ (upper limit of normal [ULN] was $8.1 \mathrm{ng} / \mathrm{mL}$ ), lactic acid dehydrogenase (LDH) was $6760 \mathrm{U} / \mathrm{L}(\mathrm{ULN} 250 \mathrm{U} / \mathrm{L})$, and $\beta$-human chorionic gonadotropin $(\beta-h C G)$ was $<5$ (normal $<5 \mathrm{IU} / \mathrm{L}$ ). Given the patient's abnormal blood counts, a bone marrow biopsy was done and revealed a moderately fibrotic marrow with an abnormal population of large, mononuclear cells with irregular contours. The overall cellularity of the marrow was 70\% from the core biopsy section (Fig. 2A). Immunostaining of the core showed that a subset of the large cells were $\mathrm{CD} 61^{+}$and had weak $\mathrm{CD} 117$ expression. By cytochemistry, these cells were negative for myeloperoxidase and periodic acidSchiff (PAS) staining with focal nonspecific esterase (NSE) positivity. Immunohistochemistry for GCT markers was negative (AFP, OCT4, placental alkaline phosphatase [PLAP], and SALL4). Overall, the CD61 immunostaining was consistent with megakaryoblasts (Fig. 2B). The hemodilute aspirate showed $15 \%$ large blasts with flow cytometry demonstrating that a subset of these blasts were $\mathrm{CD} 41^{+}, \mathrm{CD} 61^{+}$, and $\mathrm{CD} 117 \mathrm{dim}\left(\mathrm{CD} 34^{-}\right.$and $\left.\mathrm{CD} 33^{-}\right)$. Cytogenetic studies revealed a hyperdiploid karyotype: 59-69, XXY; $+X,+2,-3,-4,-6$, $-8,+10,-11,-12,-13,-16,-18,+19,+20,+21,+1-4$ mar[composite karyotype in 9/25 metaphases]/46, XY[17]. Taken together, these findings were consistent with a diagnosis of acute megakaryoblastic leukemia, or AML M7 under the former French-AmericanBritish (FAB) classification (Bennett et al. 1976).

ACT-guided core needle biopsy of the mediastinal mass showed necrotic tissue with one island of viable cartilage cells suggestive of a GCT. The patient then underwent videoassisted thoracic surgery and incisional biopsy of the mediastinal mass, which revealed necrotic fragments of tissue with scattered foci of moderately to poorly differentiated

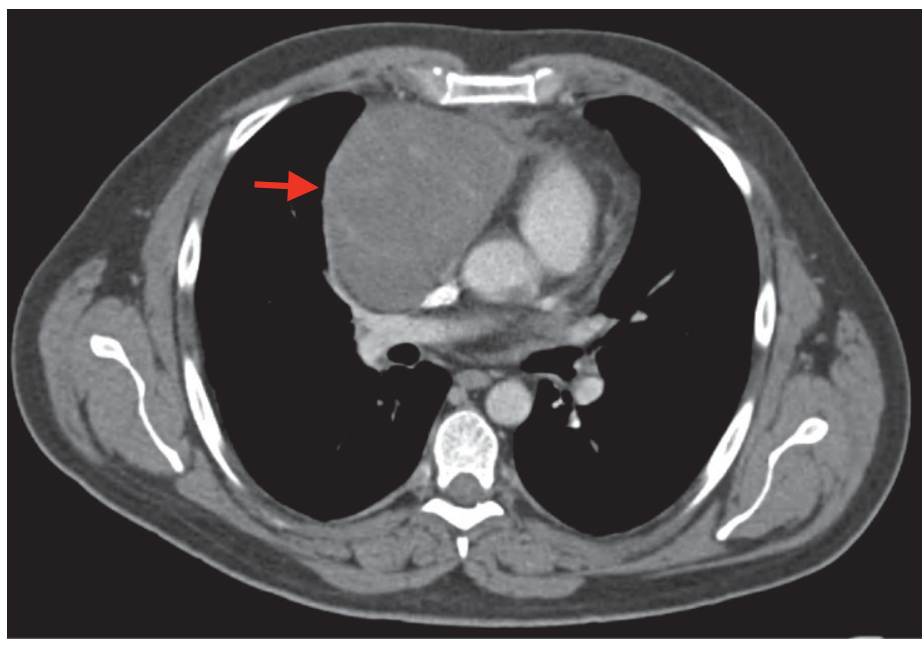

Figure 1. Computed tomography scan of the chest revealed a large, anterior mediastinal mass (red arrow). 
A

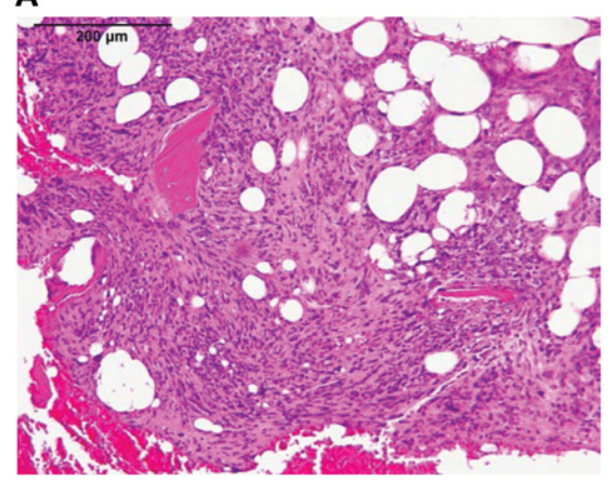

B

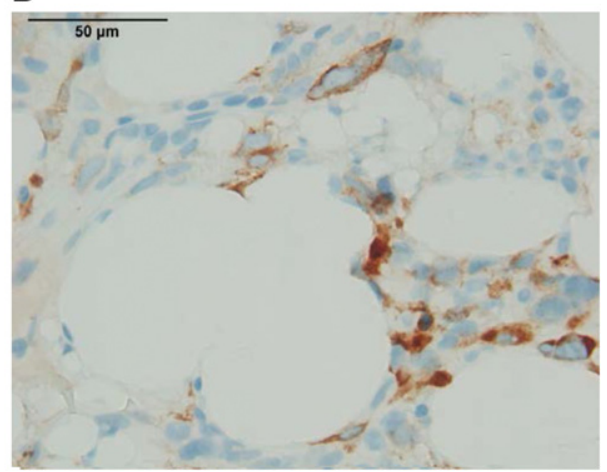

Figure 2. (A) A low-magnification (10x) view of a section from the diagnostic bone marrow biopsy shows a moderately fibrotic marrow, many infiltrating histiocytes, and an abnormal population of large, mononuclear cells. (B) A higher-magnification $(40 \times)$ view of the diagnostic bone marrow section with immunohistochemistry highlighting the $\mathrm{CD} 61^{+}$megakaryoblasts (brown staining).

adenocarcinoma (Fig. 3). Immunostaining was positive for pancytokeratin, CK7, and AFP reactivity, with nonreactivity for CK20, OCT4, SALL4, PLAP, TTF1, and napsin-A. Overall, the diagnosis was consistent with a nonseminomatous GCT. Other than the isolated island of cartilage from the first biopsy, we did not observe the presence of other germ layers. Specifically, there was no identified yolk sac component to either biopsy.

The initial pathologic diagnosis of both the AML M7 and GCT were difficult. The pathologic interpretation of the diagnostic bone marrow biopsy was complicated by marrow fibrosis and numerous background histiocytes (not uncommon in AML M7) and moderate crush artifact (Hahn et al. 2015). Although the case did not meet the World Health Organization (WHO) guidelines for a diagnosis of AML with $>20 \%$ blasts, the aspirate with $15 \%$ blasts was hemodilute, and the overall findings were consistent with a diagnosis of AML M7

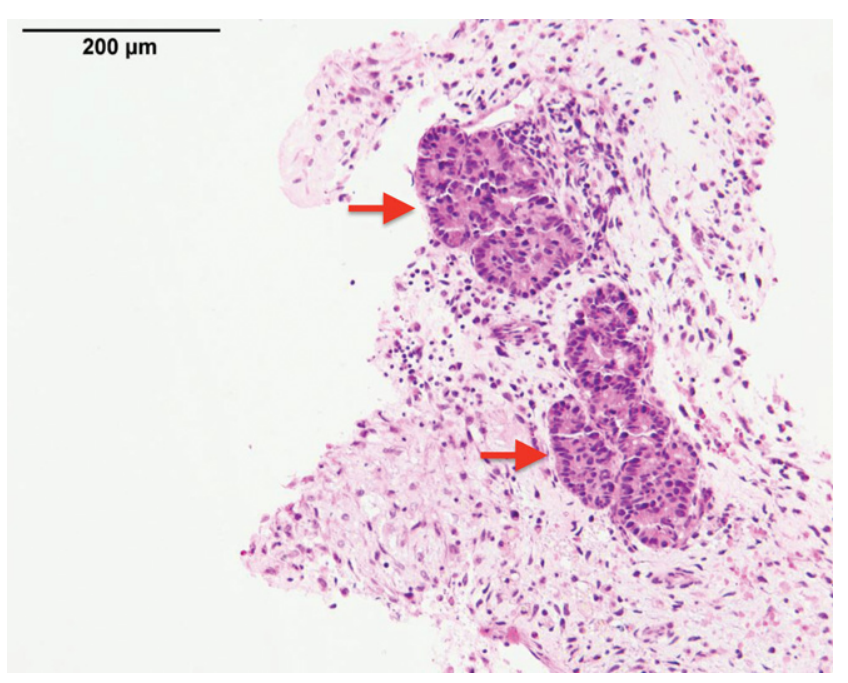

Figure 3. Low-magnification (10x) view of a section from the incisional biopsy of the mediastinal mass, which shows necrotic fragments of tissue with scattered foci of moderately to poorly differentiated adenocarcinoma (red arrows). 
COLD SPRING HARBOR Molecular Case Studies
A common clone gives rise to a GCT and AML
(Vardiman et al. 2002). Similarly, despite two biopsies, the diagnosis of the GCT was complicated by the extensive necrosis of the tumor samples. Nonetheless, it was clear that the disease in the bone marrow and the chest were two distinct malignancies. Furthermore, the issues with the suboptimal tumor samples led to unique challenges for comprehensive sequencing, which will be outlined in detail below.

The patient was initially treated with standard "7+3" AML induction chemotherapy (with cytarabine and idarubicin), and a day-15 bone marrow biopsy showed a hypocellular marrow with no evidence of leukemia. He then had a day-30 bone marrow biopsy, which showed a normocellular marrow with no evidence of leukemia. As his blood counts had recovered, he met the criteria for complete remission and went on to receive one cycle of high-dose cytarabine consolidation therapy for AML; however, shortly thereafter his GCT markers began to rise (AFP $683.8 \mathrm{ng} / \mathrm{mL}$, LDH $346 \mathrm{U} / \mathrm{L}$, and $\beta$-hCG $18 \mathrm{IU} / \mathrm{L}$ ). He was then treated with two cycles of etoposide, ifosfamide, and cisplatin (VIP regimen) for his GCT. The patient did have improvement in his tumor markers with VIP, but further restaging with imaging was not yet performed.

Fifteen days after his second cycle of VIP, he presented with nausea, vomiting, and altered mental status. A brain magnetic resonance imaging (MRI) showed evidence of marked leptomeningeal enhancement, and a lumbar puncture with cerebrospinal fluid (CSF) cytology confirmed central nervous system relapse of AML M7. The patient was treated with seven doses of intrathecal cytarabine, and his CSF cytology cleared of blasts. Unfortunately, the patient's mental status deteriorated several weeks after the completion of his intrathecal chemotherapy, and he expired 5 mo after his initial diagnosis. Although an autopsy was not performed, the cause of death was presumed to be secondary to persistent central nervous system (CNS) involvement with leukemia.

\section{Genomic Analysis}

To study the possible clonal relationship between the GCT and AML, we assayed these tumor specimens using "enhanced exome sequencing," which augments a standard exomecapture reagent with additional probes covering the coding sequences for all 264 recurrently mutated genes (RMGs) that have been identified in AML (Cancer Genome Atlas Research Network 2013; see Methods). This approach typically provides 100x median coverage over the standard exome-target regions but increases coverage of the RMG target space to an average of $\sim 400 \times-500 \times$. As noted above, the tumor samples were not optimal for sequencing studies. Therefore for the GCT, a pathologist performed laser capture microdissection on sections of the formalin-fixed paraffin-embedded (FFPE) block from the second mediastinal biopsy to isolate areas that appeared to have been viable tumor. We isolated a total of 2 ng of GCT genomic DNA (gDNA) via this method before exhausting the FFPE block. Next, as the cryopreserved cells from the diagnostic hemodilute bone marrow aspirate only contained $15 \%$ blasts, we performed flow cytometry-based cell sorting to enrich for the blasts using a gating strategy for viable $\mathrm{CD} 61^{+}$leukemic blasts. We isolated a total of $48 \mathrm{ng}$ of AML gDNA via this method. The low yields of gDNA necessitated using whole-genome amplification (WGA) on these samples to ensure that we could obtain sufficient DNA for exome-capture hybridization. We performed WGA on the entire GCT gDNA isolate (as described in the Methods) and on $8 \mathrm{ng}$ of the AML gDNA prior to library generation, capture, and sequencing. The remaining $40 \mathrm{ng}$ of AML gDNA was not amplified but used for library generation (with a unique barcode adaptor) and captured independently before sequencing. We isolated gDNA from a skin biopsy obtained at the time of the diagnostic bone marrow biopsy and sequenced as above as a "normal" comparator to define the somatic status of mutations. 
COLD SPRING HARBOR Molecular Case Studies
A common clone gives rise to a GCT and AML
A minimum of $20 \times$ coverage was achieved for $35 \%, 19 \%, 62 \%$, and $98 \%$ of the exometarget space for the GCT WGA, AML WGA, AML gDNA, and the skin gDNA, respectively. The low sequence coverage obtained using WGA libraries was typical for amplified low-input DNA samples, as was the low coverage obtained from using only 40 ng of input gDNA for the unamplified AML capture (we typically use a minimum of 150 ng of input gDNA for an exome library).

The low coverage made variant calling challenging, so in addition to our standard variant calling pipeline, we used extensive manual review and Sanger validation of putative calls. For each of the three tumor libraries, there were thousands of putative variants called, but most were low-frequency artifacts, likely resulting from the WGA and/or insufficient coverage. By limiting the analysis to protein-coding regions and focusing on data from the sequencing of the higher-quality nonamplified gDNA from the AML, we filtered the list down to 47 variants that had VAFs of $>15 \%$ in the AML gDNA sample and were putatively shared somatic events between the tumor samples. Manual review further culled this list to 29 potential variants, 10 of which we labeled "high confidence" (by manual review). We also included a PTEN mutation, despite it having low read support in the GCT sample, as it is a well-known tumor suppressor, and four other mutations that were not "high confidence" but did have supporting reads in all tumor samples (ALG1LP9, RASAL3, MT-ND6, and CRTAC1-none with established roles in cancer pathogenesis). In total, 15 mutations were chosen for Sanger validation. Out of the 15 candidate variants, seven variants were validated as somatic in both samples, two variants were validated as somatic in only the AML M7 sample, two variants were validated as germline mutations, one variant (a deletion) was not detected in any tumor library, and three were in amplicons that failed sequencing reactions. These three amplicon sequences matched multiple places on the targeted chromosomes, as it was not possible to design primers that were unique to the target. As a result, we likely generated a mixed polymerase chain reaction (PCR) product, which resulted in low-quality trace sequences (Table 1; Supplemental Fig. 1). Of the coding somatic variants that we validated, none has mutations at the identical amino acid position reported in COSMIC, other than PTEN and TP53, which do have recurrent mutations at each respective location (the mutation data were obtained from the Sanger Institute Catalogue Of Somatic Mutations In Cancer website, http://www.sanger.ac.uk/cosmic) (Bamford et al. 2004).

Of the seven somatic variants observed in both tumors, we detected a frameshift deletion in TP53 (R213fs_del) and a missense mutation in PTEN (C136R) (Fig. 4). Both mutations had VAFs approaching $100 \%$ for both samples in the exome data suggesting that both mutations were homozygous (Fig. 4C). Consistent with this observation, our analysis of the gAML M7 data is consistent with loss of heterozygosity ( $\mathrm{LOH}$ ) for genomic segments including these two genes (Supplemental Table 1). Sequence coverage for the GCT samples was very low (with 14 and two reads, respectively), so the VAF estimates are less reliable for that sample. The Sanger trace for the TP53 deletion appears homozygous in the GCT sample, but is not as clear for the PTEN mutation (Fig. 4A,B). To exclude an artifact as a consequence of WGA, we isolated $47 \mathrm{ng}$ of total genomic DNA from the first mediastinal core biopsy of the GCT (predominantly necrotic tumor) and again performed Sanger sequencing for the TP53 and PTEN alterations. The PTEN reaction failed; however, the TP53 sequencing was successful and confirmed that the deletion appeared to be homozygous.

In addition to the shared somatic variant analysis, we performed copy-number analysis using the exome-sequence data from the gAML M7 sample and detected an amplification of Chromosome 12p consistent with an isochromosome 12 (Fig. 5). As noted in the Clinical Presentation subsection, the AML karyotype from conventional cytogenetics was extremely complex with evidence of -12 in the hyperdiploid [triploid] karyotype but no evidence of isochromosome 12p (there were several marker chromosomes that were not identified, however). We were unable to perform copy-number analysis on the exome data from the GCT 


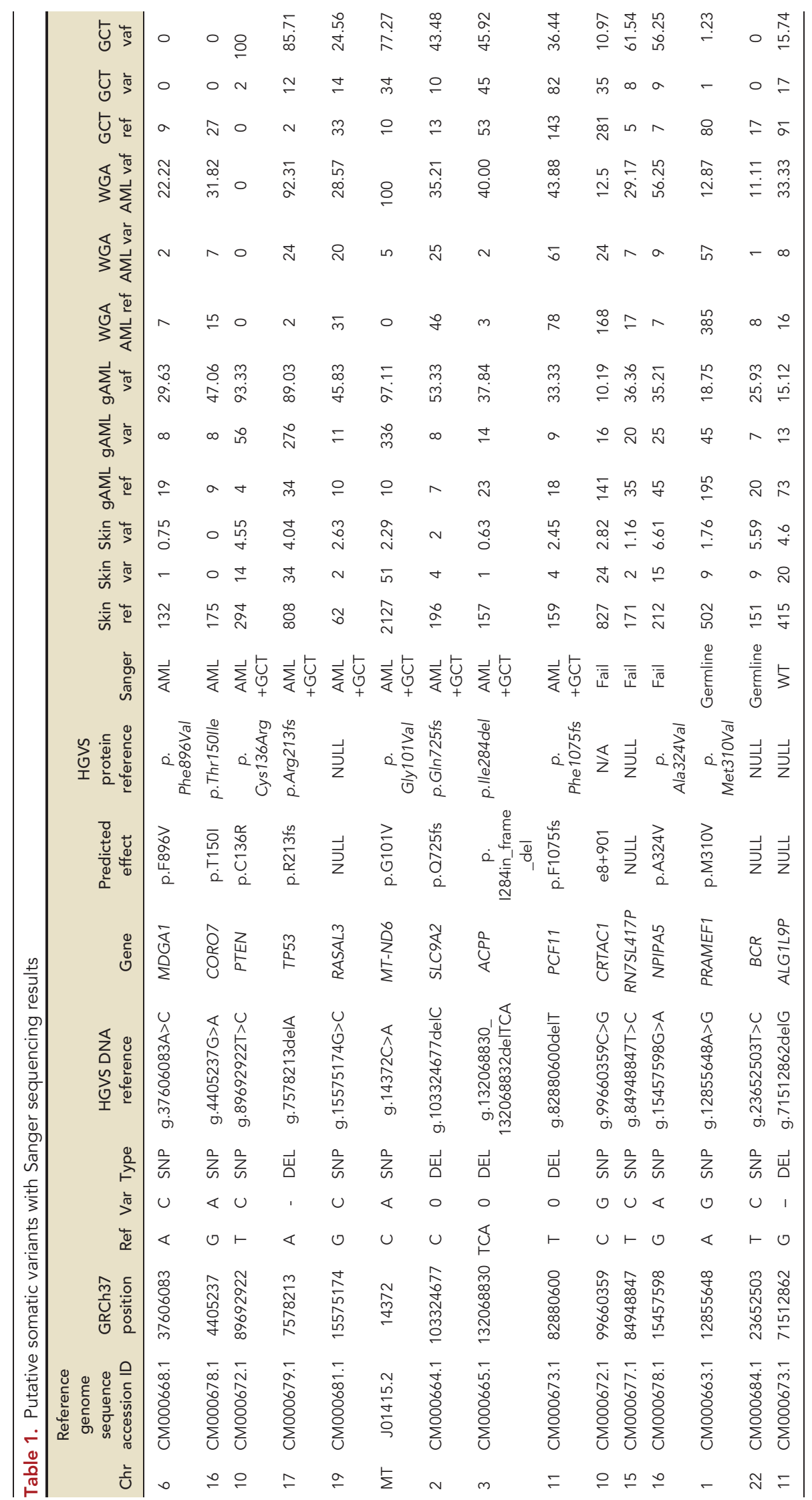



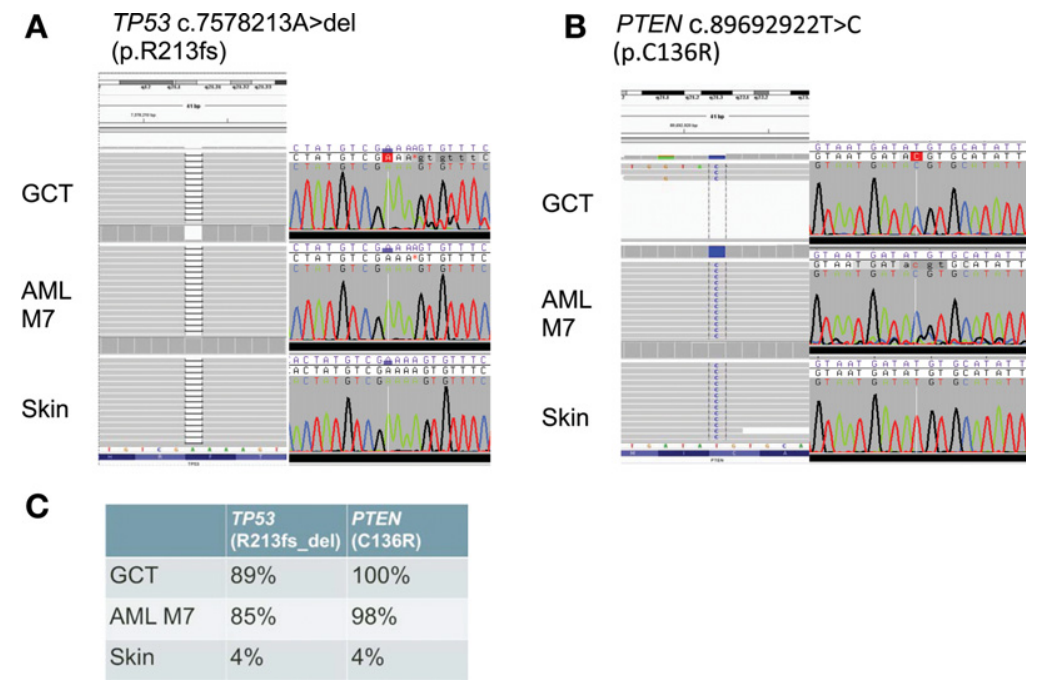

Figure 4. Shared somatic mutations identified in TP53 (A) and PTEN (B) using exome sequencing and later validated by Sanger sequencing. Variant allele fraction (VAF) of these two mutations is listed in $C$. Sanger sequencing for the PTEN and TP53 variants was also done on unamplified gDNA isolated from the necrotic GCT (germ cell tumor) sample from the first mediastinal biopsy, which also confirmed the results depicted below. AML M7, acute megakaryoblastic leukemia.

because of the low coverage. Although amplification/isochromosome of $12 p$ is the most common cytogenetic abnormality in GCTs, this feature is not common in AML (Nichols et al. 1985; Gibas et al. 1986; Bosl et al. 1989).

Finally, we performed a germline analysis on the high-quality exome data derived from the skin sample (see Supplemental Table 2). We detected a heterozygous germline variant in FANCA (S858R) that was validated by Sanger sequencing (at a VAF of 53\% with 79 reference allele reads and 88 variant allele reads; see Supplemental Fig. 2 for Sanger validation results). This mutation is known to be associated with Fanconi anemia, an autosomal-recessive

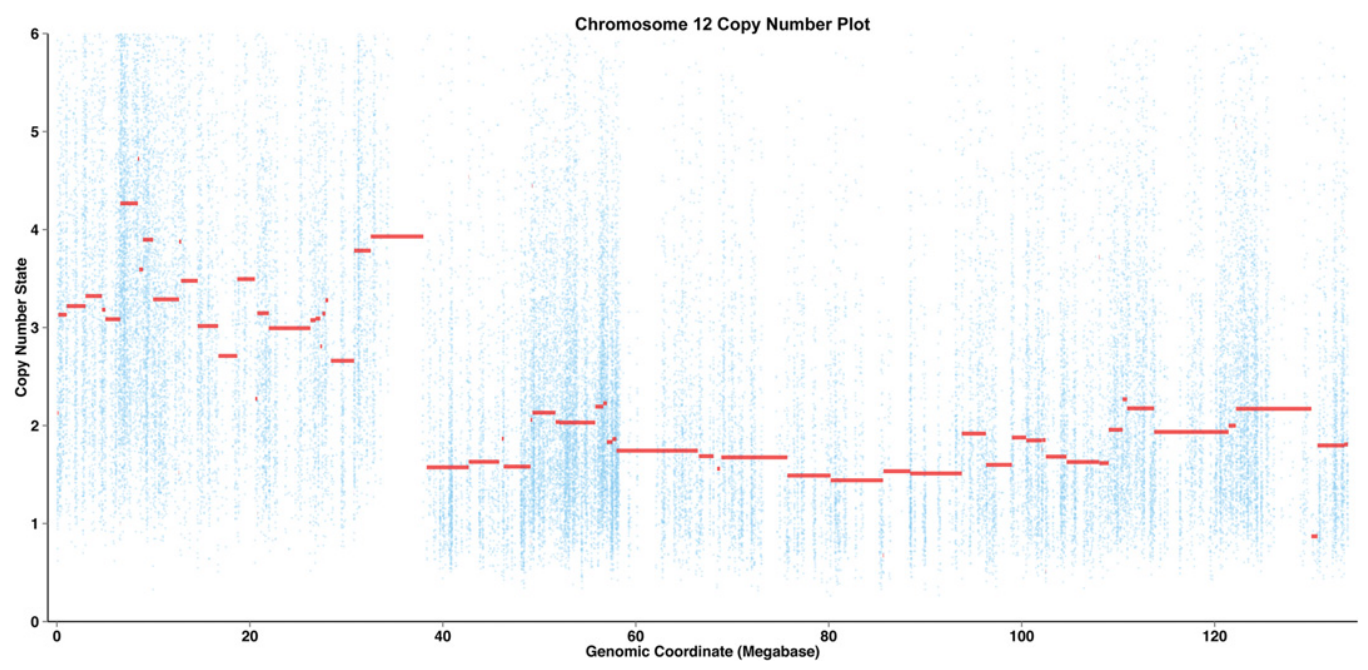

Figure 5. Copy-number analysis of the unamplified AML M7 sample demonstrated amplification of Chromosome 12p. The plot is "noisy" because of the low-sequencing coverage of the AML sample. 
disorder (Savino et al. 2003). The mutation is of uncertain significance here as it is heterozygous, and no known complementation partner was identified.

\section{DISCUSSION}

The syndrome of associated hematologic malignancies and mediastinal GCTs is extremely rare but well documented in the literature (Nichols et al. 1985; Woodruff et al. 1995; Hartmann et al. 2000; Yu et al. 2011). A nonseminomatous mediastinal GCT and an associated hematologic neoplasm that most often involves the megakaryocytic lineage of hematopoiesis characterize the syndrome. This most commonly manifests as acute megakaryoblastic leukemia (AML M7); although, it has also been associated with myelomonocytic leukemia, myelodysplasia with abnormal megakaryocytes, and essential thrombocythemia (Nichols et al. 1985). Prior reports observed a median time of $\sim 6$ mo from the diagnosis of the GCT to diagnosis of the hematologic disease, with rare reports of a synchronous presentation, as seen in our patient (Nichols et al. 1985; Hartmann et al. 2000). Generally, this syndrome must be distinguished from a primary malignancy with a therapyrelated secondary malignancy (i.e., a primary GCT and then a secondary AML, which typically develops at least a year following exposure to cytotoxic chemotherapy), but that potential scenario is not a complicating factor in this case (Nichols et al. 1985; Yu et al. 2011).

Isolated de novo AML M7 is also very rare in adults, only accounting for $<1 \%$ of all AML cases. Outcomes for patients with isolated AML M7 are very poor, with a median overall survival reported between $\sim 4$ and 10 mo (Hahn et al. 2015). Similarly, a primary mediastinal nonseminomatous GCT is also a rare cancer, because extragonadal GCTs account for only $<5 \%$ of GCTs in males. Primary mediastinal nonseminomatous GCTs (in the absence of an associated hematologic malignancy) also have a poor prognosis when compared with testicular (or extragonadal retroperitoneal) nonseminomatous GCTs with a 5 -yr overall survival rate of only $45 \%$ (Bokemeyer et al. 2002).

The hematologic malignancies associated with primary mediastinal GCTs typically behave aggressively, with patients frequently succumbing to direct effects or complications of the hematologic malignancy, either before initiation of therapy or after achieving a shortlived response to treatment. The prognosis for these patients is extremely poor, with a median overall survival of only $\sim 5$ mo (Hartmann et al. 2000). Allogeneic stem cell transplant represents the only curative approach for patients, but few patients are able to move forward to transplantation (Hiramatsu et al. 2008).

Multiple theories have surfaced in an attempt to explain the connection between the GCT and AML. Previous reports have proposed that these malignancies originated from a common, early (i.e., mesodermal) stem cell progenitor, developing separately along germ cell and hematopoietic lines and resulting in the GCT and hematologic malignancy, respectively (Lee 1994). Alternatively, others have argued that the syndrome resulted from the differentiation of a transformed germ cell into the hematopoietic lineage (Bosl et al. 1989; Landanyi et al. 1990; Orazi et al. 1993). This proposed clonal relationship was initially established by the demonstration of isochromosome 12p in both GCT and hematologic malignancy specimens (Nichols et al. 1985; Hartmann et al. 2000; Yu et al. 2011). However, a later study presented evidence for hematopoietic cells within the yolk sac component of GCTs that morphologically resembled the leukemic blasts found in the bone marrow of four patients, suggesting that leukemia developed from a differentiated component of the GCT that subsequently "metastasized" to the bone marrow to cause leukemia (Orazi et al. 1993). Despite these findings, further elucidation of the genomic link between these malignancies has been slow to develop, on account of their rarity (Nichols et al. 1985, 1990; Chaganti et al. 1989; Woodruff et al. 1995; Yu et al. 2011). It should again be noted that 
COLD SPRING HARBOR Molecular Case Studies
A common clone gives rise to a GCT and AML we did not identify a yolk sac component from the patient's GCT biopsies, which may have been a result of the extensive amount of necrosis present in these samples. This is one limitation of the current study in terms of defining the specific cell of origin of these two related tumors.

More recently, Oshrine et al. (2014) demonstrated shared mutations in TP53 (L130P missense) and PTEN (G251V) in a male patient with concurrent AML M7 and a mediastinal nonseminomatous GCT. The patient also had evidence of isochromosome 12p in both cancers. In our case, enhanced exome sequencing was carried out on the AML M7 and GCT samples, and we identified common somatic mutations in TP53 (R213fs_del) and PTEN (C136R missense), which were confirmed through Sanger sequencing. Importantly, we validated five additional shared mutations between the AML M7 and the GCT (in RASAL3, MT-ND6, SLC9A2, ACPP, and PCF11). Although the significance of any of these particular mutations to tumorigenesis is unknown (none are recurrently mutated in $A M L$ ), the characterization of a total of seven shared somatic variants between the GCT and AML M7 firmly establishes the presence of a shared founding clone between the two distinct malignancies. Furthermore, as demonstrated in numerous previous reports, we were able to identify an amplification of Chromosome 12p in the AML M7, further supporting a common clonal ancestry. Unfortunately, we were not able to perform a copy-number analysis on the GCT given the data quality, and the limited coverage of the gAML precluded a detailed copy-number analysis, which is already difficult with exome data. An improved copy-number analysis of both the GCT and AML would have added power to our study and may have made it possible to infer the clonal evolution of the cancers. Moreover, as this was exome data, we also do not have information on structural variation, which should be recognized as another limitation of the current work.

We identified two variants that were specific to the AML M7 sample (in MDGA1 and CORO7). Again, these variants have no clear link to AML leukemogenesis and are probably passenger mutations. It is also possible that they do not represent subclonal mutations that are specific to the AML sample-they may not have been detected in the GCT sample because of limitations associated with sequencing a WGA sample, including low sequence coverage and amplification-induced bias (Pinard et al. 2006). Other than the TP53 mutation, we did not identify any other mutation in a gene that is known to be recurrently mutated in AML.

Germline analysis revealed a heterozygous germline variant in FANCA (S858R). The S858R mutation is known to be associated with Fanconi anemia, which is an autosomalrecessive disorder (Savino et al. 2003). The mutation is of uncertain significance in our patient, because it is heterozygous with no known complementation partner identified. It should be noted, however, that Oshrine et al. also found a heterozygous germline mutation of unknown significance, an ATM D1080E missense mutation, in their patient. Although involved in different pathways, both genes play pivotal roles in DNA damage repair.

PTEN mutations and isochromosome 12p are exceedingly rare in AML. We identified no PTEN mutations in the 200 cases of AML sequenced in the TCGA project (Cancer Genome Atlas Research Network 2013). Isochromosome 12p alterations are also as uncommon in AML, as previously shown in multiple studies (see Nichols et al. 1990). Conversely, TP53 mutations are relatively rare in GCTs (Masters and Köberle 2003). We now report the second case to describe the association between shared TP53 and PTEN mutations, as well as an alteration in Chromosome 12p, in the syndrome of concurrent GCT and AML M7. The mutual occurrence of a mutation in both PTEN and TP53 has not been previously reported in AML, other than the current study and the study by Oshrine et al. (2014).

In summary, our data provide some of the strongest evidence to date that the tumors in this syndrome arise from a shared founding clone (i.e., a single cell ultimately evolved into both tumors). Given the limitations of our data, we could not deduce the clonal evolution 
COLD SPRING HARBOR Molecular Case Studies
A common clone gives rise to a GCT and AML of the GCT and AML, however. More specifically, we could not determine whether the AML was a subclone of the GCT, which will require further study. Although speculative, mutations in both TP53 and PTEN, along with isochromosome 12p, may represent the common genomic abnormalities that drive this rare syndrome that is distinct from the typical pathogenesis of either isolated AML or typical GCTs. Inactivation of TP53 may partially explain the poor prognosis of these patients, because it is a known factor in chemoresistance and in unfavorable risk AML. Additional effort should be focused on the pathogenesis of this syndrome, because we have not been able to clarify the cell of origin. However, our results have significant implications for future genomic screening and on developing novel targeted treatment approaches for this rare patient population with an extremely poor prognosis.

\section{METHODS}

For the GCT, we extracted 2 ng of gDNA from laser capture microdissected tumor on the FFPE block of the incisional mediastinal biopsy. Briefly, tumor cells from hematoxylin and eosin-stained slides were identified by a pathologist (I.S.H.) and captured from 10- $\mu$ m stained sections using an Arcturus PixCell instrument. The captured cells were then microdissected into the cap of $500-\mu \mathrm{L}$ safe-lock tubes filled with $50 \mu \mathrm{L}$ ALT buffer (QIAGEN). DNA extraction was performed as previously described (Cancer Genome Atlas Research Network 2013). WGA was performed using the Rubicon Genomics PicoPLEX WGA kit according to the manufacturer's instructions. We split the sample into three separate aliquots before amplification and then pooled these samples before sequencing to minimize the risk of bias introduced by the WGA. For the AML sample, 48 ng of gDNA was isolated from flow-sorted megakaryoblasts from cryopreserved cells banked during the diagnostic bone marrow biopsy. We performed flow sorting using a BD Bioscience FACSAria II cell sorter with a gating strategy for $\mathrm{CD} 1^{+}$positive cells (CD45dim/low side scatter blasts that were $\mathrm{CD} 3^{-}, \mathrm{CD}_{3}{ }^{-}$, and $\mathrm{CD} 61^{+}$). All antibodies were from BD Bioscience. For microscopy, we used an Olympus BX41 microscope with an Olympus DP72 camera.

Eight nanograms of the isolated AML gDNA was used for WGA libraries with the Rubicon Genomics PicoPLEX kit and the remaining 40 ng of unamplified gDNA was used for separate exome capture. For enhanced exome sequencing, we used the Roche NimbleGen SeqCap EZ Human Exome Library v3.0 kit plus a spike-in with probes covering all coding exons of significantly recurrently mutated genes in AML (from Integrated DNA Technologies, 264 genes, with the addition of the TERT promoter and WT1 noncoding regions) (Cancer Genome Atlas Research Network 2013; Klco et al. 2015). The four dual-indexed libraries were then pooled and run on a single flow cell of an Illumina HiSeq 2500 sequencer in rapid run mode.

Sanger sequencing was done as previously described (Ellis et al. 2012) on the WGA AML and GCT DNA samples. In addition, we isolated 47 ng of gDNA (unamplified) from the core biopsy FFPE block of the first mediastinal GCT biopsy for Sanger sequencing for the TP53 and PTEN mutations.

Exome-sequence data were aligned to reference sequence build GRCh37-lite-build37 using BWA-MEM version 0.7.7 (parameters: -t 4 -q 5::) (Li and Durbin 2009). Data were then merged and deduplicated using picard version 1.113 (https://broadinstitute.github.io/ picard/). We detected somatic single-nucleotide variants (SNVs) using the union of three callers: (1) SAMtools version $r 982$ (parameters: mpileup-BuDS) (Li et al. 2009) filtered by snp-filter version v1 and false-positive-filter v1 (parameters: -bam-readcount-version 0.4 -bam-readcount-min-base-quality 15 -max-mm-qualsum-diff 100) and intersected with Somatic Sniper version 1.0.4 (parameters: -F vcf -q 1 -O 15) (Larson et al. 2012) filtered by false-positive version v1 (parameters: -bam-readcount-version 0.4 -bam-readcount-min-base-quality 


\begin{tabular}{lcccc}
\hline \multicolumn{2}{l}{ Table 2. Exome-sequencing coverage metrics } \\
\hline Sample & $\begin{array}{c}\text { Number of } \\
\text { reads }\end{array}$ & $\begin{array}{c}\text { Percentage of } \\
\text { duplicate reads }\end{array}$ & $\begin{array}{c}\text { Percentage of } \\
\text { reads mapped }\end{array}$ & $\begin{array}{c}\text { On target mean } \\
\text { coverage }\end{array}$ \\
\hline Skin & $195,524,973$ & 6.89 & 99.88 & 183.94 \\
gDNA AML M7 & $50,171,683$ & 6.07 & 99.88 & 38.11 \\
WGA AML M7 & $44,068,667$ & 4.1 & 99.3 & 17.66 \\
WGA GCT & $120,320,947$ & 3.13 & 99.55 & 36.50 \\
\hline
\end{tabular}

gDNA, genomic DNA; AML M7, acute megakaryoblastic leukemia; WGA, whole-genome amplification; GCT, germ cell tumor.

15) then somatic-score-mapping-quality version v1 (parameters: -min-mapping-quality 40 -min-somatic-score 40); (2) VarScan 2.3.6 (Koboldt et al. 2012) filtered by varscan-highconfidence version $\mathrm{v} 1$ then false-positive version $\mathrm{v} 1$ (parameters: -bam-readcount-version 0.4 -bam-readcount-min-base-quality 15); and (3) Strelka version 1.0.11 (parameters: isSkipDepthFilters = 1) (Saunders et al. 2012) (see Table 2 for coverage metrics).

We detected somatic indels using the union of four callers: (1) gatk-somatic-indel version 5336 (McKenna et al. 2010) filtered by false-indel version v1 (parameters: -bam-readcountversion 0.4 -bam-readcount-min-base-quality 15); (2) pindel version 0.5 (Ye et al. 2009) filtered by pindel-somatic-calls version v1 then pindel-vaf-filter version v1 (parameters: -variant-freq-cutoff $=0.08$ ) then pindel-read-support version v1; (3) VarScan 2.3.6 (parameters: -nobaq -version r982) (Koboldt et al. 2012) filtered by varscan-high-confidence-indel version v1; and (4) Strelka version 1.0.11 (parameters: isSkipDepthFilters = 1) (Saunders et al. 2012). Transcript annotation was performed using Ensembl v74_37. All somatic variants were manually reviewed using Integrative Genomics Viewer (IGV) (Robinson et al. 2011).

Somatic copy-number variants (CNVs) were called and recentered from the aligned BAM file using VarScan2 (http://dkoboldt.github.io/varscan) (Koboldt et al. 2012) using default parameters. Segments of copy number were identified using circular binary segmentation as implemented in DNAcopy for R (Seshan VE and Olshen A. DNAcopy: DNA copy-number data analysis $R$ package, version 1.40.0). VarScan2 was also used in conjunction with the DNAcopy package to identify regions of copy-number-neutral $\mathrm{LOH}$. For a list of all called CNVs from the gDNA AML sample, please see Supplemental Table 3.

Germline SNPs and indels were detected in the skin sample using SAMtools version r982 (parameters: -nobaq -version r982:-min-coverage 3 -min-var-freq 0.20 -p-value 0.10 -strand-filter 1 -map-quality 10) filtered by false-positive v1 (parameters -bam-readcountmin-base-quality: 15 -bam-readcount-version: 0.4 -max-mm-qualsum-diff: 100). These were unioned with SNPs called with SAMtools version r962 filtered by var-filter-snv v1, then false-positive-vcf v1 (parameters: -bam-readcount-min-base-quality: 15 -bam-readcount-version: 0.4 -max-mm-qualsum-diff: 100). Variants were filtered against an internally curated list of cancer-related genes, and then further curated to retain those likely to have functional consequences (Supplemental Table 2).

\section{ADDITIONAL INFORMATION}

\section{Ethics Statement}

The patient was enrolled in a single-institution, tissue-banking protocol approved by the human studies committee at Washington University. He provided written informed consent for comprehensive sequencing studies, including exome sequencing. The Washington University Institutional Review Board (IRB) approved this protocol. 
Competing Interest Statement

The authors have declared no competing interest.

Received August 28, 2015; accepted in revised form October 22, 2015

\section{Database Deposition and Access}

The sequence data for all tumors and matched normal samples has been deposited in the $\mathrm{NCBI}$ database of Genotypes and Phenotypes (dbGaP; http://www.ncbi.nlm.nih.gov/gap) under accession number: phs000159.v8.p4. PTEN and TP53 variants have been deposited in ClinVar (http://www.ncbi.nlm.nih.gov/clinvar/) under accession numbers SCV000257331SCV000257340.

\section{Acknowledgments}

This work was done as part of the Washington University School of Medicine Genomics Tumor Board, which is a collaborative effort between the Division of Oncology and the McDonnell Genome Institute. The authors thank Drs. John F. Dipersio, Timothy J. Ley, Elaine R. Mardis, and Richard K. Wilson for their supervision, mentorship, and support of this project.

\section{Author Contributions}

C.L. analyzed data and wrote the manuscript. P.R. conceived parts of the study and wrote the manuscript. C.A.M. analyzed data and wrote the manuscript. I.S.H. performed experiments. P.W. conceived parts of the study. B.A.O. analyzed the data and wrote the manuscript. M.O., V.M., and R.T.D. performed experiments. E.J.D. performed experiments and wrote the manuscript. M.G., O.L.G, and L.D.W. conceived parts of the study, analyzed the data, and wrote the manuscript.

\section{Funding}

The research was funded with private research support from the Division of Oncology and the McDonnell Genome Institute. L.D.W. is supported by the National Institutes of Health/National Cancer Institute (NIH/NCI) K08 CA166229. O.L.G. is supported by NIH/ $\mathrm{NCl}$ K22 CA188163.

\section{REFERENCES}

Bamford S, Dawson E, Forbes S, Clements J, Pettett R, Dogan A, Flanagan A, Teague J, Futreal PA Stratton MR, et al. 2004. The COSMIC (Catalogue of Somatic Mutations in Cancer) database and website. Br J Cancer 91: 355-358.

Bennett JM, Catovsky D, Daniel MT, Flandrin G, Galton DA, Gralnick HR, Sultan C. 1976. Proposals for the classification of the acute leukaemias. French-American-British (FAB) co-operative group. Br J Haematol 33: 451-458.

Bokemeyer C, Nichols CR, Droz J, Schmoll H, Horwich A, Gerl A, Fossa SD, Beyer J, Pont J, Kanz L, et al. 2002. Extragonadal germ cell tumors of the mediastinum and retroperitoneum: results from an international analysis. J Clin Oncol 20: 1864-1873.

Bosl GJ, Dmitrovsky E, Reuter VE, Samaniego F, Rodriguez E, Geller NL, Chaganti RS. 1989. Isochromosomes of chromosome 12: clinically useful marker for male germ cell tumors. J Natl Cancer Inst 84: 1874-1878.

Cancer Genome Atlas Research Network. 2013. Genomic and epigenomic landscapes of adult de novo acute myeloid leukemia. N Engl J Med 368: 2059-2074.

Chaganti RS, Ladanyi M, Samaniego F, Offit K, Reuter VE, Jhanwar SC, Bosl GJ. 1989. Leukemic differentiation of a mediastinal germ cell tumor. Genes Chromosomes Cancer 1: 83-87.

DeMent SH, Eggleston SC, Spivac JL. 1985. Association between mediastinal germ cell tumors and hematologic malignancies: report of two cases and review of the literature. Am J Surg Pathol 9: 23-30.

Ellis MJ, Ding L, Shen D, Luo J, Suman V, Wallis J, Van Tine BA, Hoog J, Goiffon R, Goldstein TC, et al. 2012. Whole genome analysis informs breast cancer response to aromatase inhibition. Nature 486: 353-360.

Gibas Z, Prout GR, Pontes JE, Sandberg AA. 1986. Chromosome changes in germ cell tumors of the testis. Cancer Genet Cytogenet 19: 245-252. 
Hahn AW, Li B, Prouet P, Giri S, Pathak R, Martin MG. 2015. Acute megakaryocytic leukemia: what have we learned. Blood Rev doi: 10.1016/j.blre.2015.07.005.

Hartmann JT, Nichols CR, Droz JP, Horwich A, Gerl A, Fossa SD, Beyer J, Pont J, Fizazi K, Einhorn L, et al. 2000. Hematologic disorders associated with primary mediastinal nonseminomatous germ cell tumors. $J$ Natl Cancer Inst 92: 54-61.

Hiramatsu H, Morishima T, Nakanishi H, Mizushima Y, Miyazaki M, Matsubara H, Kobayashi M, Nakahata T, Adachi S. 2008. Successful treatment of a patient with Klinefelter's syndrome complicated by mediastinal germ cell tumor and AML(M7). Bone Marrow Transplant 41: 907-908.

Ikdahl T, Josefsen D, Jakobsen E, Delabie J, Fossa SD. 2007. Concurrent mediastinal germ-cell tumour and haematological malignancy: case report and short review of literature. Acta Oncol 47: 466-469.

Klco JM, Miller CA, Griffith M, Petti A, Spencer DH, Ketkar-Kulkarni S, Wartman LD, Christopher M, Lamprecht TL, Helton NM, et al. 2015. Association between mutation clearance after induction therapy and outcomes in acute myeloid leukemia. JAMA 314: 811-822.

Koboldt DC, Zhang Q, Larson DE, Shen D, McLellan MD, Lin L, Miller CA, Mardis ER, Ding L, Wilson RK. 2012. VarScan 2: somatic mutation and copy number alteration discovery in cancer by exome sequencing. Genome Res 22: 568-576.

Landanyi M, Samaniego F, Reuter VE, Motzer RJ, Jhanwar SC, Bosl GJ, Chaganti RS. 1990. Cytogenetic and immunohistochemical evidence for the germ cell origin of a subset of acute leukemias associated with mediastinal germ cell tumors. J Natl Cancer Inst 82: 221-227.

Larson DE, Harris CC, Chen K, Koboldt DC, Abbott TE, Dooling DJ, Ley TJ, Mardis ER, Wilson RK, Ding L. 2012. SomaticSniper: identification of somatic point mutations in whole genome sequencing data. Bioinformatics 28: 311-317.

Lee KC. 1994. Hematopoietic precursor cells within the yolk sac tumor component are the source of secondary hematopoietic malignancies in patients with mediastinal germ cell tumors. Cancer 73: 1535.

Li H, Durbin R. 2009. Fast and accurate short read alignment with Burrows-Wheeler transform. Bioinformatics 25: 1754-1760.

Li H, Handsaker B, Wysoker A, Fennell T, Ruan J, Homer N, Marth G, Abecasis G, Durbin R; 1000 Genome Project Data Processing Subgroup. 2009. The sequence alignment/map format and SAMtools. Bioinformatics 25: 2078-2079.

Masters JR, Köberle B. 2003. Curing metastatic cancer: lessons from testicular germ-cell tumours. Nat Rev Cancer 3: 517-525.

McKenna A, Hanna M, Banks E, Sivachenko A, Cibulskis K, Kernytsky A, Garimella K, Altshuler D, Gabriel S, Daly M, et al. 2010. The genome analysis toolkit: a MapReduce framework for analyzing next-generation DNA sequencing data. Genome Res 20: 1297-1303.

Medvinsky A, Dzierzak E. 1996. Definitive hematopoiesis is autonomously initiated by the AGM region. Cell 86: 897-906.

Nichols CR, Hoffman R, Einhorn LH, Williams SD, Wheeler LA, Garnick MB. 1985. Hematologic malignancies associated with primary mediastinal germ-cell tumors. Ann Intern Med 102: 603-609.

Nichols CR, Roth BJ, Heerema N, Griep J, Tricot G. 1990. Hematologic neoplasia associated with primary mediastinal germ-cell tumors. N Engl J Med 322: 1425-1429.

Orazi A, Neiman RS, Ulbright TM, Heerema NA, John K, Nichols CR. 1993. Hematopoietic precursor cells within the yolk sac tumor component are the source of secondary hematopoietic malignancies in patients with mediastinal germ cell tumors. Cancer 71: 3873-3881.

Oshrine BR, Olsen MN, Heneghan M, Wertheim G, Daber R, Wilmoth DM, Biegel JA, Pawel B, Aplenc R, King RL. 2014. Acquired isochromosome 12p, somatic TP53 and PTEN mutations, and a germline ATM variant in an adolescent male with concurrent acute megakaryoblastic leukemia and mediastinal germ cell tumor. Cancer Genet 207: 153-159.

Pinard R, de Winter A, Sarkis GJ, Gerstein MB, Tartaro KR, Plant RN, Egholm M, Rothberg JM, Leamon JH. 2006. Assessment of whole genome amplification-induced bias through high-throughput, massively parallel whole genome sequencing. BMC Genomics 7: 216.

Robinson JT, Thorvaldsdóttir H, Winckler W, Guttman M, Lander ES, Getz G, Mesirov JP. 2011. Integrative genomics viewer. Nat Biotechnol 29: 24-26.

Saunders CT, Wong W, Swamy S, Becq J, Murray LJ, Cheetham RK. 2012. Strelka: accurate somatic small-variant calling from sequenced tumor-normal sample pairs. Bioinformatics 28: 1811-1817.

Savino M, Borriello A, D'Apolito M, Ciscuolo M, Del Vecchio M, Bianco AM, Di Perna M, Calzone R, Nobili B, Zatterale A, et al. 2003. Spectrum of FANCA mutations in Italian Fanconi anemia patients: identification of six novel alleles and phenotypic characterization of the S858R variant. Hum Mutat 4: 338-339.

Vardiman JW, Harris NL, Brunning RD. 2002. The World Health Organization (WHO) classification of the myleooid neoplasms. Blood 100: 2292-2302.

Woodruff K, Wang N, May W, Adrone E, Denny C, Feig SA. 1995. The clonal nature of mediastinal germ cell tumors and acute myelogenous leukemia. Cancer Genet Cytogenet 79: 25-31. 
Ye K, Schulz MH, Long Q, Apweiler R, Ning Z. 2009. Pindel: a pattern growth approach to detect breakpoints of large deletions and medium sized insertions from paired-end short reads. Bioinformatics 25: 2865-2871.

Yu N, Kim HR, Cha YJ, Park EK, Kim JW. 2011. Development of acute megakaryoblastic leukemia with isochromosome (12p) after a primary mediastinal germ cell tumor in Korea. J Korean Med Sci 26: 1099-1102.

Zhao GQ, Dowell JE. 2012. Hematologic malignancies associated with germ cell tumors. Expert Rev Hematol 5: 427-437. 


\section{COLD SPRING HARBOR Molecular Case Studies}

\section{A common founding clone with TP53 and PTEN mutations gives rise to a concurrent germ cell tumor and acute megakaryoblastic leukemia}

Charles Lu, Peter Riedell, Christopher A. Miller, et al.

Cold Spring Harb Mol Case Stud 2016, 2: a000687

Access the most recent version at doi: $10.1101 / \mathrm{mcs} . a 000687$

$\underset{\text { Material }}{\operatorname{Supplementary}} \quad{ }_{1}^{\mathrm{http}: / / \text { molecularcasestudies.cshlp.org/content/suppl/2015/12/11/2.1.a000687.DC }}$

References This article cites 36 articles, 4 of which can be accessed free at:

http://molecularcasestudies.cshlp.org/content/2/1/a000687.full.html\#ref-list-1

License This article is distributed under the terms of the Creative Commons

Attribution-NonCommercial License, which permits reuse and redistribution, except for commercial purposes, provided that the original author and source are credited.

Email Alerting Receive free email alerts when new articles cite this article - sign up in the box at the Service top right corner of the article or click here. 